\title{
Large-Area Phase-Contrast X-ray Imaging System Using a Two-Crystal X-ray Interferometer -Development of an Interference-Pattern-Based Feedback Positioning System-
}

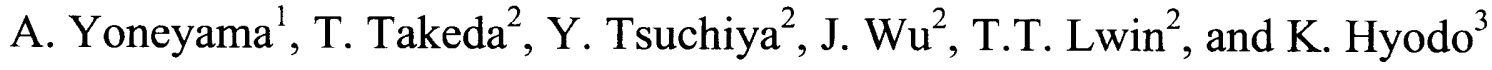 \\ ${ }^{\prime}$ Advanced Research Laboratory, Hitach Ltd , Hatoyama, Saltama 350-0395, ${ }^{2}$ Institute of Clinical Medicine, \\ University of Tsukuba, Ibarakı 305-8575, Japan, and ${ }^{3}$ Institute of Materials Science, High Energy Accelerator \\ Research Organization, Tsukuba, Ibarakı 305-0801, Japan
}

\begin{abstract}
To more accurately suppress the phase fluctuation of the interference beams of a large-area phase-contrast Xray imaging system using a two-crystal X-ray interferometer, a new feedback positionıng system (FPS) has been developed and applied The motion of interference patterns replaces the intensity of the interference beam in a small region as the feedback signal used in controlling the rotation of crystal blocks relative to each other This FPS kept the phase fluctuation of the interference beams within $\pi / 15$ over more than six hours Examples of high-quality two and three-dımensional ımages of biological samples obtained by the imaging system with the new FPS are given
\end{abstract}

\section{INTRODUCTION}

Phase-contrast X-ray ımagıng usıng X-ray interferometer has strong potentıal as a biomedical imaging technique The phase-shift cross-sections for light elements are about 1000 times greater than the absorption cross section [1,2], so the phase-contrast technique gives us a method for the fine observation of biomedical samples that requires neither the use of contrast agents nor harmful levels of X-ray exposure This high sensitivity technique was implemented by monolithic triple Laue-case (LLL) X-ray interferometer [3], and used in the radiographic observation of small pieces of rat cerebellum and human metastasized liver tumor tissue [1,2] In addition, this imaging technique has been combined with computerized tomography in a technology for three-dimensional observation (phase-contrast CT [4]), which has been used to observe small columnar pieces of tissue from various organs and to distinguish cancerous from normal tissue [5-7]

Larger fields of view and suppression of the thermal disturbance caused by the sample's heat are two prerequisites to the wider biomedical application of this imaging technique However, the monolithic X-ray interferometer is incapable of meeting these requirements, and therefore it is required to divide the interferometer into two crystal blocks

Operation of the skew-symmetric two-crystal X-ray interferometer (STXI) [8] requires the precise control of only two rotational axes This structure thus has an advantage over other possible forms of two-crystal interferometer, which require more complex control [8] With this advantage in mind, the authors have been developing a large-area phase-contrast X-ray imaging system based on the STXI [9-12] The latest system has a $60 \times 30-\mathrm{mm}$ field of view, and has been used to observe the phase maps (spatial distributions of phase shift caused by samples) of several biomedical samples [12] In the present study, the authors have developed a new feedbackpositioning system that more accurately stabilizes the phase fluctuation of the interference beams, and successfully used in the three-dimensional observation of biological samples

\section{IMAGING SYSTEM WITH A NEW FEEDBACK-POSITIONING SYSTEM}

Figure 1 gives a schematıc view of the imaging system and newly developed feedback positioning system (FPS) The incident X-ray is horizontally expanded by the asymmetric crystal, and then input to the STXI The STXI 
generates two interference beams, one for CCD-based imaging detector 1 [13] used in measuring the phase-maps and the other for detector 2 used in the operation of the new FPS

The STXI was positioned by the mechanical positioning system composed of three tables S1 for adjusting the STXI under the Bragg condition against the incident X-rays, S2 and the tilt tables for tuning the $\theta$ and $\rho$ rotation between the crystal blocks, respectively To attain the required sub-nanoradian mechanical stability around $\theta$ axis for the operation of the STXI, the rigidity of table S2 was improved by using of a sleeve bearing In addition, a fine adjustment mechanism driven by a lamınated piezoelectric actuator (PZT) was installed These measures together achieved a minımum incremental rotation (step size) of $006 \mathrm{nrad}$ for table S2

The new FPS was installed to more accurately stabilize the phase fluctuation of the interference beams, which was caused by the drift rotation around the $\theta$ axis Taking advantage of the fact that the drift rotation is reflected in motion of the interference pattern, we use the following procedure in the new FPS

1 Before commencing feedback operation, detect a region of the interference pattern for storage as the initial pattern, $I o$

2 Start the feedback operation

3 Detect the interference pattern $I$ which is currently in the same region

4 Calculate the pattern shift $d x$ between $I$ and $I o$

5 Using $d x$, calculate the voltage $V$ that the high-precision voltage source must apply to the PZT so that motion of the interference pattern is cancelled out

6 Apply $V$ to the PZT

7 Return to 3

In our previous positioning system [10], the intensity of the interference beam in a small region was used as the feedback signal This approach was incapable of distinguishing the effects of drift rotation from other factors that affect the intensity, such as positional drift of the monochromator's crystal The new FPS has eliminated this shortcoming, and enabled the more accurate control over longer periods with the above method

Imaging detector 2 (for feedback operation) was composed of a scintillator $\left(\mathrm{Gd}_{2} \mathrm{O}_{2} \mathrm{~S} 30\right.$ microns thick), mirror, lens system, and CCD camera The input X-ray image was converted to visible light image by the scintillator, reflected in the mirror (this prevents X-ray-induced damage to the lens system), and then focused on the CCD camera by the lens system To avoid the generation of any mechanical vibration by the CCD camera, a fan-free water-cooled model (Photonic Science FDI $1300 \times 1024$ pixels, 68 microns square, 6 fps for 12-bit digitization) with an electronic shutter was used

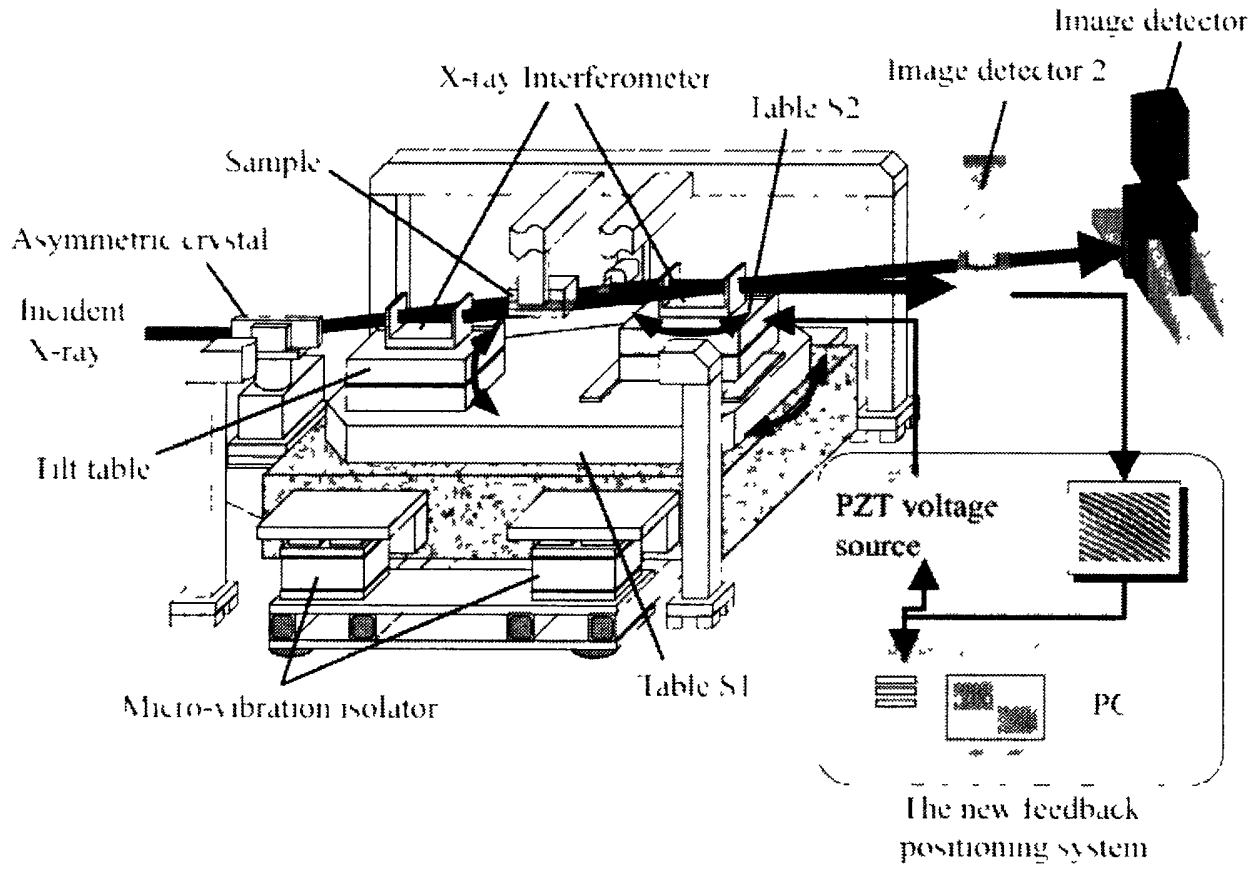

FIGURE 1. A schematıc view of the imaging system and newly developed feedback positıonıng system 


\section{RESULTS AND DISCUSSION}

Performance evaluation of the new FPS and demonstrative observation of biological samples by the whole system were carried out at the beam-line BL-14C1 of the Photon Factory in Tsukuba, Japan The vertically fanshaped beam emitted from a vertical wiggler was monochromated to $177 \mathrm{keV}$ by a $\mathrm{Si}(220)$ double-crystal monochromator and then input to the imaging system

Figure 2 depicts the operation of the new FPS over time The black lines indicate the phase fluctuations calculated from the pattern shift $d x$ and the gray line indicates the voltage $V$ applied to the PZT of table S2 The images in the chart show the interference patterns (in a region $03-\mathrm{mm}$ wide and $12-\mathrm{mm}$ high) every two hours, while the interference pattern was constantly recorded with the same period as the exposure time The exposure time for detection of the interference pattern was $2 \mathrm{~s}$, and the feedback system's time constant was set at $5 \mathrm{~s}$ The phase fluctuation was effectively suppressed by the changes in voltage over the roughly six-hour period shown here The standard deviation of the phase fluctuation was $\pi / 15$, which corresponds to a drift rotation of $006 \mathrm{nrad}$

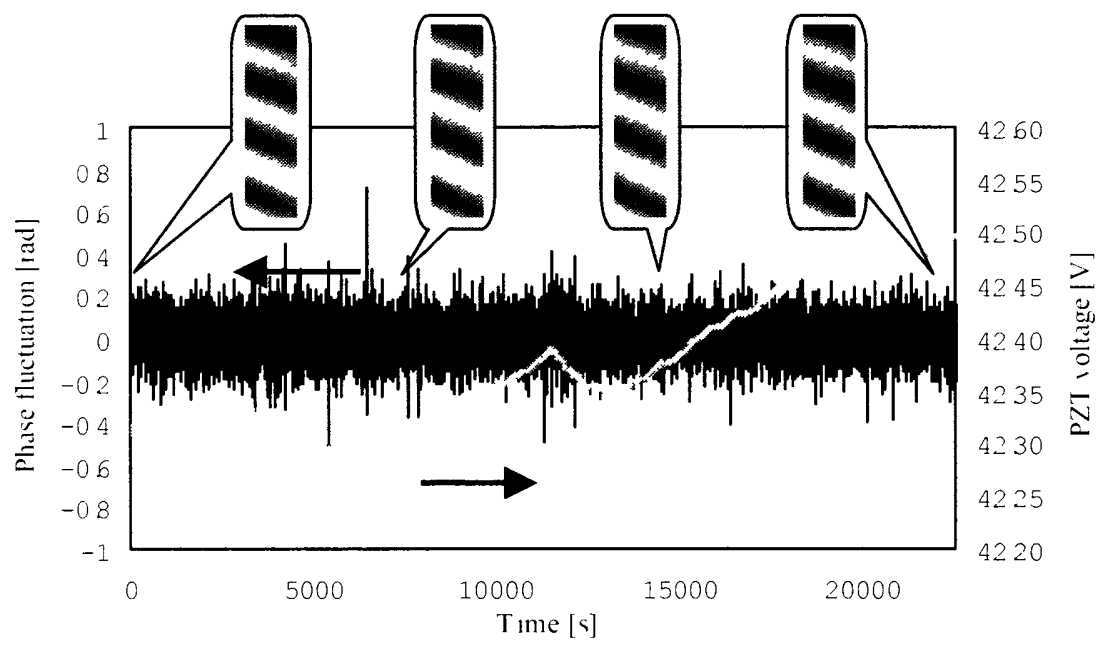

FIGURE 2. Chart over tıme of the pattern shift $d x$ and of the voltage $V$ applied to the PZT of table S2

Figure 3 shows a phase map of the liver from a rabbit with VX2 cancer obtained by the fringe scanning method with subtraction of the background phase [12] The number of fringe scans was set to five, with a 10-s exposure to obtain each of the interference patterns The image is size of $33-\mathrm{mm}$ wide and $30-\mathrm{mm}$ high Since cancerous and normal tissue produce different phase shifts, the tissue types were clearly distinguishable from each other In addition, the blood vessel bordering the cancerous tissue appeared as a relatıvely bright region

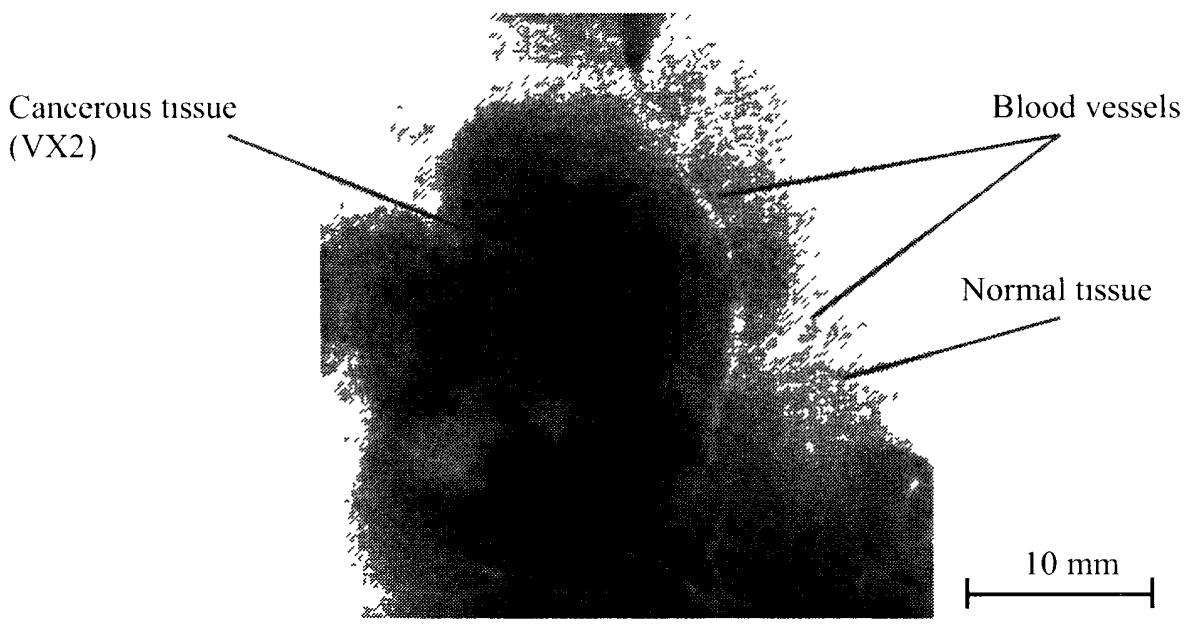

FIGURE 3. Phase map of the liver from a rabbit with VX2 cancer 
Figure 4 shows a three-dimensional image of a columnar piece of liver from a rabbit with VX2 cancer To obtain this image, the sample was placed in a water-filled sample cell and rotated in 072 -degree steps (250 projections) A phase map for each projection was obtained by same process as was used to obtain Fig 3, except that the exposure tıme for the detection of each interference pattern was $5 \mathrm{~s}$ To reconstruct the tomograms, filtered back-projection with a Shepp-Logan filter was used The sample was $10 \mathrm{~mm}$ in diameter and 5-mm high As in Fig 3, the regions of cancerous and normal tissue are clearly distinguishable from each other, and the blood vessels are clearly visible

These experiments were approved by the Medical Committee for the Use of Anımals in Research of the University of Tsukuba, and it conformed to the guidelines of the American Physiological Society

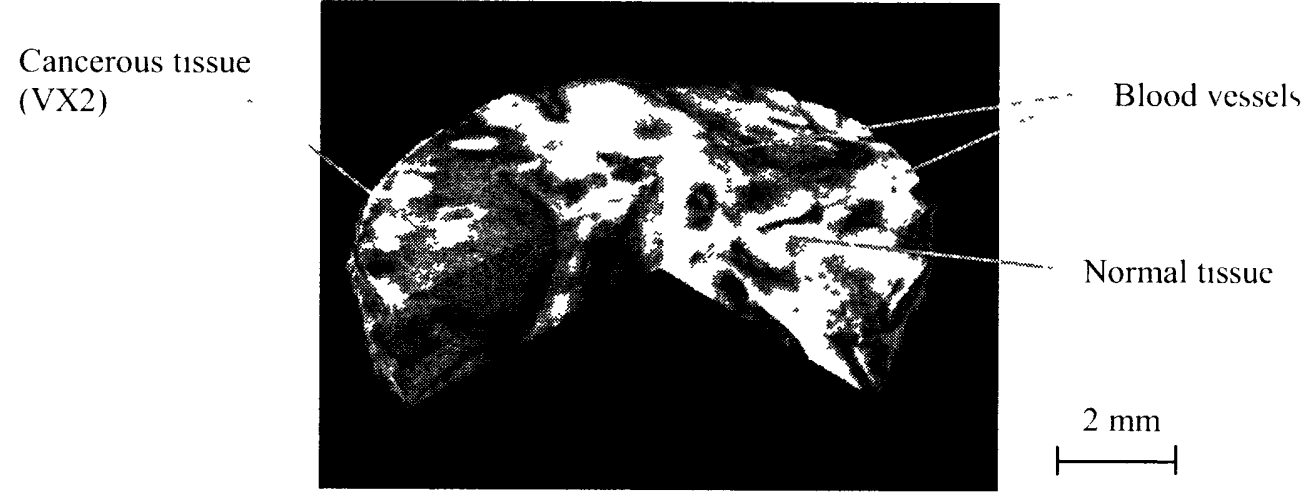

FIGURE 4. A thee-dimensional image of a columnar piece of liver from a rabbit with VX2 cancer

\section{SUMMARY}

A feedback positioning system has been newly developed to more accurately suppress the phase fluctuation of the interference beams of the large-area phase-contrast X-ray imaging system using two-crystal X-ray interferometer The phase fluctuation was thus suppressed to within $\pi / 15$ over more than six hours The effectiveness of the imaging system with this feedback positioning system was demonstrated by a phase map and three-dimensional image of a rabbit liver with VX2 cancer These results show the accuracy and stability of this imaging system in sample observation, since it is already applicable to the observation of large and in vivo biological samples, the system is approaching broad application in the field of biomedical observation

This study was carried out with the support through Special Coordination Funds of the Ministry of Education, Culture, Sports, Science and Technology of the Japanese Government and under Proposal No 2002S2-001 approved by the High Energy Accelerator Research Organization

\section{REFERENCES}

1 Momose, A, and Fukuda, J, Med Phys 22, 375-380(1995)

2 Takeda, T, Momose, A, Ital, Y, Wu, I, and Hirano, K, Acad Radıol 2, 799-803(1995)

3 Bonse, U, and Hart, M, Appl Phys Lett 6, 155-156(1965)

4 Momose, A, Nucl Instrum Meth A352, 622-628(1995)

5 Momose, A, Takeda, T, Ita1, Y, and Hirano, K, Nature Med 2, 473-475(1996)

6 Beckmann, F , Bonse, U , Busch, F, and Gunnewig, O, J Comp Assist Tomogr 21, 539-553(1997)

7 Takeda, T, Momose, A, Hirano, K, Haraoka, S, Watanabe, T , and Ita1, Y, Radiology 214, 298-301(2000)

8 Becker, P, and Bonse, U, J Appl Cryst 7, 593-598(1974)

9 Momose, A, Yoneyama, A, and Hirano, K, J Synchrotron Rad 4, 311-312(1997)

10 Yoneyama, A, Momose, A, Seya, E, Hirano, K, Takeda, T, and Ita1, Y, Rev Sc1 Instrum 70, 4582-4586(1999)

11 Yoneyama, A, Momose, A, Koyama, I, Seya, E, Takeda, T, Ita1, Y, Hirano, K, and Hyodo, K, J Synchrotron Rad 9, 277-281(2002)

12 Yoneyama, A, Takeda, T, Tsuchıya, Y, Wu, J, Lwin, T T, Koızumı, A, Hyodo, K, and Ita1, Y, Nucl Instrum Meth (submitted)

13 Momose, A, Takeda, T, Yoneyama, A, Koyama, I, and Ital, Y, Nucl Instrum Meth A 467, 917-920(2001) 